MSUTH 92/05

February, 1993

revised

\title{
Studying the Top Quark via the $W$-Gluon Fusion Process
}

\author{
Douglas O. Carlson AND C.-P. Yuan \\ Department of Physics and Astronomy \\ Michigan State University \\ East Lansing, MI 48824
}

\begin{abstract}
We show that studying the single top quark production via the $W$-gluon fusion process can provide unique information on: $(i)$ the measurement of the decay width $\Gamma\left(t \rightarrow b W^{+}\right)$; (ii) probing the symmetry breaking mechanism by measuring the form factor of $t-b-W$; (iii) testing the Effective- $W$ Approximation prior to supercolliders; $(i v)$ testing $\mathrm{CP}$ violation by observing different production rates of $\bar{p} p \rightarrow t X$ and $\bar{p} p \rightarrow \bar{t} X ;(v)$ testing $\mathrm{CP}$ violation from the almost hundred percent longitudinally polarized top quark decay; in addition to the measurement of the top quark mass. In contrast to the recent claim by R. K. Ellis and S. Parke, we show that this process is extremely useful at the Tevatron.
\end{abstract}




\section{Introduction}

The top quark has been found to be heavier than $45 \mathrm{GeV}$ from SLAC and LEP experiments and $91 \mathrm{GeV}$ from $\mathrm{CDF}$ data. The first limit is model independent while the second limit is for the Standard Model (SM) top quark. Since the top quark is heavy, of the same order of magnitude as the $W$-boson mass, any physical observable related to the top quark may be sensitive to new physics. The top quarks will therefore allow many new tests of the SM and new probes of physics at the $100 \mathrm{GeV}$ scale.

In the past, one of us proposed a method, via the $W$-gluon fusion process $q g\left(W^{+} g\right) \rightarrow t \bar{b} X$, to detect a heavy top quark at hadron colliders. ${ }^{[1,2]}$ We showed that an almost perfect efficiency for the "kinematic $b$ tagging" can be achieved due to the characteristic features of the transverse momentum and rapidity distributions of the spectator quark which emitted the virtual $W$. For a $140 \mathrm{GeV}$ SM top quark, the production rate $(\sim 4 \mathrm{pb})$ for the $W$-gluon fusion process $W g \rightarrow(t \bar{b}+\bar{t} b)$ is about a factor of 4 smaller than the usual QCD processes $q \bar{q}, g g \rightarrow t \bar{t}$ at the Tevatron. However, the $W$-gluon fusion process becomes more important for a heavier top quark.

With a $100 \mathrm{pb}^{-1}$ luminosity at the Tevatron, it is important to re-examine this process to see whether it can teach us something which is either impossible or difficult to be learned from studying the usual QCD process. Both CDF and D0 are upgrading detection efficiencies for forward jets, the coverage of leptons, and the efficiency of tagging $b$ jets in their detectors. Putting all of these together, we show that studying this process can give a better measurement on the decay width $\Gamma(t \rightarrow$ $\left.b W^{+}\right)$of the top quark. For instance, the top quark decay width $\Gamma\left(t \rightarrow b W^{+}\right)$can be measured better via this process at the Tevatron than the usual QCD process at the supercolliders by almost an order of magnitude for a $140 \mathrm{GeV}$ top quark. ${ }^{[6]}$ Therefore, it allows probing the symmetry breaking mechanism by measuring the form factors of $t-b-W$ from its production rate. This process also offers a chance to study the "Effective- $W$ Approximation, ${ }^{,[5]}$ which is essential in studying the 
strongly interacting longitudinal $W$ system, prior to the time of the future highenergy colliders SSC and LHC. At the future hadron-hadron, electron-hadron, or $e^{-} e^{+}$colliders, one might be able to use similar processes ${ }^{[3]}$ to measure the Cabbibo-Kobayashi-Maskawa matrix element $\left|V_{t s}\right|$ due to the copious production of the top quark. ${ }^{[4]}$

It is important to examine different types of operators the $t-b-W$ vertex might have. ${ }^{[3]}$ For instance, one should examine the form factors which result from an effective lagrangian obtained by higher order QCD and electroweak effects. One may also examine the form factors to test the plausibility of having the nonuniversal gauge couplings of $t-b-W$ due to some dynamical symmetry breaking scenario. ${ }^{[7]}$

The most important consequence of a heavy top quark is that to a good approximation it decays as a free quark since its lifetime is short and it does not have time to bind with light quarks before it decays. Thus we can use the polarization properties of the top quark as additional observables to test the SM and to probe new physics. Furthermore, because the heavy top quark has the weak two-body decay $t \rightarrow b W^{+}$, it will analyze its own polarization.

Top quarks will have longitudinal polarization if weak effects are present in their production. In the SM, the heavy top quark produced via the $W$-gluon fusion process is left-handed polarized, and unpolarized from the usual QCD process, at the Born level. With the large production rate expected for top quarks at the SSC and the LHC, it will be possible to make a detailed study of the interactions of the top quark including polarization effects. If new interactions occur, they may manifest themselves in an enhancement of the polarization effects in the production of the top quark via the $W$-gluon fusion process. ${ }^{[3]}$ Furthermore, if CP is violated, the production rate of $t$ from $\bar{p} p\left(W^{+} g\right) \rightarrow t \bar{b} X$ would be different from that of $\bar{t}$ from $\bar{p} p\left(W^{-} g\right) \rightarrow \bar{t} b X$. Therefore, one can detect large CP violation effects by observing the difference in the production rates of $t$ and $\bar{t}$.

In the $W$-gluon fusion process the top quark is almost hundred percent longitudinally polarized. This allows us to probe $\mathrm{CP}$ violation in the decay process 
$t \rightarrow W^{+} b \rightarrow l^{+} \nu_{l} b$. The obvious observable for this purpose is ${ }^{[8]}$ the expectation value of the time-reversal quantity $\overrightarrow{\sigma_{\mathbf{t}}} \cdot\left(\widehat{\mathbf{p}_{b}} \times \widehat{\mathbf{p}}_{l}\right)$ where $\overrightarrow{\sigma_{\mathbf{t}}}$ is the polarization vector of $t$, and $\widehat{\mathbf{p}}_{b}\left(\widehat{\mathbf{p}}_{l}\right)$ is the unit vector of the $b\left(l^{+}\right)$momentum in the rest frame of the top quark. This was suggested in Ref. 3 and further studied in Ref. 9.

In contrast to the recent claim by R. K. Ellis and S. Parke, ${ }^{[10]}$ we show in the next section that this process is extremely useful at the Tevatron. With kinematic cuts, there are about $20 t$ or $\bar{t}$ (including the branching ratio $W \rightarrow e$, or $\mu$ ) from this process produced at the Tevatron with a $100 \mathrm{pb}^{-1}$ luminosity and $\sqrt{S}=1.8$ $\mathrm{TeV}$. The major background is the $W+$ jets event which is of the same order as the signal event with $b$-tagging using a vertex detector. The $b$-quark tagging efficiency is assumed to be $100 \%$ with no misidentifications.

\section{Brief Results}

In this letter, we will only briefly report our results and leave the details to future publications. For simplicity, we only discuss $140 \mathrm{GeV}$ top quark production at the Tevatron.

Since the single top quark produced from the $W$-gluon fusion process involves a very important and not yet well-developed technique of handling the kinematics of a heavy $b$ parton inside a hadron, ${ }^{[11]}$ the event rate of single top quark production via this process is estimated by using the method proposed in Ref. 12. The prescription proposed in Ref. 12 was shown to be correct in leading log approximation for the total production rate of $t$ in the $W$-gluon fusion process. A study on how to implement a similar technique when kinematic cuts are applied in the event analysis is in progress. ${ }^{[11]}$ We chose the QCD scale to be $M_{W}$, the $W$-boson mass, and found that the total production rate is $2 \mathrm{pb}$ for $t$ and the same rate for $\bar{t}$ at the Tevatron. The parton distribution function used in our calculation is the leading order set, Fit SL, of Morfin-Tung. ${ }^{[13]}$ For comparison, the total production rate of $t \bar{t}$ via the usual QCD process is about $16 \mathrm{pb}$. 
In Ref. 14, we showed that the intrinsic width of the top quark can not be measured at the SSC and the LHC through the usual QCD process. For instance, the intrinsic width of a $140 \mathrm{GeV}$ Standard Model top quark is about $0.6 \mathrm{GeV}$, and the full width at half maximum of the reconstructed top quark invariant mass is about $11 \mathrm{GeV}$ after including the detector resolution effects by smearing the final state parton momenta. A similar conclusion was also given from a hadron level analysis. ${ }^{[15]}$ Can the top quark width $\Gamma\left(t \rightarrow b W^{+}\right)$be measured better than the factor $11 / 0.6 \sim 20$ mentioned above? The answer is yes. As pointed out in Ref. 14, the width $\Gamma\left(t \rightarrow b W^{+}\right)$can be measured by counting the production rate of top quarks from the $W-b$ fusion process which is equivalent to the $W$-gluon fusion process by a proper treatment of the bottom quark and the $W$ boson as partons inside the hadron. ${ }^{[11]}$ The $W$-boson which interacts with the $b$-quark to produce the top quark can be treated as an on-shell boson in the leading log approximation. ${ }^{[5]}$ The moral is that even under the approximations considered, a factor of 2 uncertainty in the production rate for this process gives a factor of 2 uncertainty in the measurement of $\Gamma\left(t \rightarrow b W^{+}\right)^{[16]}$ This is still much better than what can be done at the SSC and the LHC through the usual QCD process. Therefore, this is an extremely important measurement to be made at the Tevatron because it directly tests the coupling of $t-b$ - $W$ at the tree level.

The Effective- $W$ Approximation has been the essential tool used in studying the strongly interacting longitudinal $W$ system to probe the symmetry breaking sector at the SSC. One can learn about the validity of the Effective- $W$ Approximation prior to supercolliders by studying the $W$-gluon fusion process at the Tevatron.

For heavy top quark production, the dominant subprocess is $W_{L} b \rightarrow t$, where $W_{L}$ is a longitudinally polarized $W$-boson. Based on the argument of the electroweak chiral lagrangian, ${ }^{[17]}$ it is likely that new physics will show up in the interaction of a longitudinal $W$, which is equivalent to a Goldstone boson characterizing the symmetry breaking mechanism, and a heavy fermion. Hence, measuring the form factor of $t-b-W$ in this process provides a probe on the symmetry breaking 
sector.

As discussed above, the top quark produced from this process is about one hundred percent polarized due to the left-handed charged current in the SM. This allows a test of $\mathrm{CP}$ violation asymmetry. ${ }^{[3]}$ A detailed analysis using this process testing the CP violation asymmetry in the decay of $t$ was performed in Ref. 9. Furthermore, if the CP violating effects in the couplings of $t-b-W$ are large, the production rates of $\bar{p} p \rightarrow t X$ and $\bar{p} p \rightarrow \bar{t} X$ from the $W$-gluon fusion process will be significantly different.

To show that a $140 \mathrm{GeV}$ top quark produced from this process can be detected at the Tevatron, we performed a Monte Carlo study on the $W+2$ jets mode of the signal,

$$
q^{\prime} b \rightarrow q t\left(\rightarrow b W^{+}\left(\rightarrow l^{+} \nu\right)\right)
$$

with $l^{+}=e^{+}$or $\mu^{+}$. After the kinematic cuts

$$
\begin{array}{ll}
P_{T}^{q}>15 \mathrm{GeV}, & \left|\eta^{q}\right|<3.5, \\
P_{T}^{l}>15 \mathrm{GeV}, & \left|\eta^{l}\right|<2, \\
P_{T}^{b}>40 \mathrm{GeV}, & \left|\eta^{b}\right|<2, \\
E_{T}>15 \mathrm{GeV}, & \Delta R_{q b}>0.7,
\end{array}
$$

the signal rate is about $0.11 \mathrm{pb}$. It is important to note that the typical rapidity of the spectator jet $(q)$ in the signal event is about 1.6 at the Tevatron. ${ }^{[1]}$ The distribution of $\eta^{q}$ is asymmetric because the Tevatron is a $\bar{p} p$ collider. A cut on $\left|\eta^{q}\right|<3.5$ keeps almost all the signal events. An asymmetric cut on $P_{T}$ was used to suppress the major EW-QCD background process $W+2$ jets.

The $t \bar{t}$ background is not important after vetoing the events with $\geq 3$ jets. $^{[10]}$ We require one charged lepton and two jets, one of which is a $b$ or $\bar{b}$, to pass the kinematic cuts (2.2). The semileptonic decay mode of the $t \bar{t}$ background process $t \bar{t} \rightarrow b \bar{b} l^{+} l^{\prime-} \nu \bar{\nu}^{\prime}$ then gives a rate of $\sim 0.014 \mathrm{pb}$, where $l^{+}$includes both $e^{+}$and $\mu^{+}$, and $l^{\prime-}$ includes $e^{-}, \mu^{-}$and $\tau^{-}$. This is about a factor of 8 smaller than the 
signal rate. ${ }^{[19]}$ The event rate after the cuts (2.2) for one of the top quarks ( $t$ or $\bar{t}$ ) decaying semileptonically and the other hadronically is smaller than the purely semileptonic decay of $t \bar{t}$ by a factor of 20 .

Following the method proposed in Ref. 1, the longitudinal momentum $P_{z}^{\nu}$ of the reconstructed (fake) neutrino in $t \bar{t}$ events can be determined by requiring the invariant mass of the charged lepton and the reconstructed (fake) neutrino to be the mass of the $W$-boson. If a physical solution cannot be found, this event is discarded. As expected, because the missing transverse momentum of the $t \bar{t}$ event is in general not that of a real neutrino from $W$ decay, neither the transverse mass $M_{T}^{e \nu b}$ nor the top invariant mass $m_{t}$ distribution look like those of the signal event even if a physical solution exists for $P_{z}^{\nu}$.

Hence, the dominant background is

$$
u \bar{d}, c \bar{s} \rightarrow b \bar{b} W^{+}\left(\rightarrow l^{+} \nu\right)
$$

After the kinematic cuts (2.2), this background rate is about 1.3 times the signal rate. The other backgrounds such as $c g \rightarrow b g W^{+}$are suppressed due to the small CKM matrix element $\left|V_{t s}\right| \sim 0.03$ to 0.05 .

Because the top quark produced from the signal process is left-handed polarized, $l^{+}$tends to move against the moving direction of the top quark in the center-of-mass frame of $q$ and $t^{[3,18]}$ However, in the background event, the distribution of $\cos \theta_{l q}$ is almost flat after the cuts $(2.2)$. $\left(\theta_{l q}=\pi-\theta_{l}\right.$, where $\theta_{l}$ is the the polar angle of $l^{+}$in the rest frame of $t$ defined in the $q t$ center-of-mass frame.) We can further improve the signal-to-background ratio by imposing

$$
\cos \theta_{l q}>-0.4
$$

This was performed by using the constructed $P_{z}^{\nu}$ information to boost into the center-of-mass of the system, we then boost into the rest frame of the top. After cut (2.4), the signal-to-background ratio is about one. There are $\sim 10$ signal 
events (2.1) with a $100 \mathrm{pb}^{-1}$ luminosity and $\sqrt{S}=1.8 \mathrm{TeV}$. The signal event rate was reduced by a factor of 3 after applying the cuts (2.2) and (2.4) on the process (2.1). However, we anticipate the signal event rates will be somewhat larger than the ones reported here after properly treating the $b$ quark as a parton inside the proton. ${ }^{[20]}$ In Fig. 1, we show the reconstructed invariant mass $\left(m_{t}\right)$ of the top quark. The method of reconstructing $m_{t}$ was already presented in Ref. 1. We therefore conclude that the top quark can be detected and studied via this process at the Tevatron.

To incorporate the effects of detector efficiencies, we smear the final state parton momentum using a Gaussian distribution with

$$
(\Delta E / E)_{l}=15 \% / \sqrt{E}, \quad \text { and } \quad(\Delta E / E)_{q, b}=50 \% / \sqrt{E} .
$$

The $m_{t}$ distribution becomes slightly broader as shown in Fig. 2. However, both the signal and the background rates are almost the same as those in Fig. 1 obtained with a perfect detector.

In our future publications, we will show various distributions of the signal and background events to illustrate that the signal and the background events can be further distinguished. However, we do not suggest imposing additional cuts. Instead, we propose other methods such as using neural networks ${ }^{[21]}$ to obtain an even cleaner signal. After including both charged states, we conclude that about 20 signal events produced from the $W$-gluon fusion process can be detected and studied to test the Standard Model and probe new physics at the Tevatron. ${ }^{[22]}$ In reality, the efficiency of $b$-tagging is not perfect. A $50 \%$ efficiency of $b$-tagging gives a total of $\sim 10$ signal events with $100 \mathrm{pb}^{-1}$ integrated luminosity and $\sqrt{S}=1.8$ $\mathrm{TeV}$. On the other hand, the Tevatron might upgrade to $\sqrt{S}=3.6 \mathrm{TeV}$ with higher luminosity $\sim 2 \mathrm{fb}^{-1}$. This amounts to a factor of 150 increase in the signal rate. Hence, both D0 and CDF should make efforts to study the top quark produced via the $W$-gluon fusion process. 
Acknowledgements:

C.P.Y. would like to thank J. Collins, G. Kane, G. Ladinsky, S. Mrenna and Y.-P. Yao for collaboration. To them and S. Errede, J. Gunion, S. Parke, M. E. Peskin and M. Veltman, we are grateful for discussions. This work was funded in part by the TNRLC grant \#RGFY9240.

\section{REFERENCES}

1. C.-P. Yuan, Phys. Rev. D41 (1990) 42.

2. S. Dawson, Nucl. Phys. B249, 42 (1985); S. Dawson and S. S. D.Willenbrock, 284, 449 (1987); S. S. D. Willenbrock and D. A. Dicus, Phys. Rev. D 34, 155 (1986); F. Anselmo, B. van Eijk and G. Bordes, Phys. Rev. D 45, 2312 (1992); T. Moers, R. Priem, D. Rein and H. Reithler in Proceedings of Large Hadron Collider Workshop, preprint CERN 90-10, 1990.

3. G. L. Kane, G. A. Ladinsky and C.-P. Yuan, Phys. Rev. D45 (1992) 124.

4. D. Carlson and C.-P. Yuan, in preparation.

5. For a review, see S. Cortese and R. Petronzi, Phys. Lett. B276 (1992) 203.

6. The measurement of $\Gamma\left(t \rightarrow b W^{+}\right)$is equivalent to the measurement of $t-b-W$ couplings.

7. R.D. Peccei and X. Zhang, Nucl. Phys. B337 (1990) 269.

8. J.D. Jackson, S.B. Treiman and H.W. Wyld, Jr., Phys. Rev. 106 (1957) 517;

R.B. Curtis and R.R. Lewis, Phys. Rev. 107 (1957) 543.

9. B. Grzadkowski and J.F. Gunion, preprint UCD-92-7, 1992.

10. R.K. Ellis and S. Parke, Phys. Rev. D46 (1992) 3785.

11. J. C. Collins and C.-P. Yuan, in preparation.

12. J. C. Collins and Wu-Ki Tung, Nucl. Phys. B278 (1986) 934; F. Olness and Wu-Ki Tung, Nucl. Phys. B308 (1988) 813; M. Aivazis, F. Olness and Wu-Ki Tung, Phys. Rev. Lett. 65 (1990) 2339. 
13. J. Morfin and Wu-Ki Tung, Z. Phys. C52 (1991) 13.

14. S. Mrenna and C.-P. Yuan, Phys. Rev. D46 (1992) 1007.

15. SDC technical Design Report, preprint SDC-92-201, 1992. It was concluded that the top quark invariant mass has a width of $9 \mathrm{GeV}$ for a $150 \mathrm{GeV}$ top quark.

16. After the top quark is found, one can measure the branching ratio of $t \rightarrow b W^{+}\left(\rightarrow l^{+} \nu\right)$ by the ratio of $(2 l+j e t s)$ and $(1 l+j e t s)$ event rates from $t \bar{t}$ production. Then, a model independent measurement of the decay width $\Gamma\left(t \rightarrow b W^{+}\right)$can be made by counting the production rate of $t$ in the $W$-gluon fusion process.

17. S. Weinberg, Phys. Rev. 166 (1968) 1568; S. Coleman, J. Wess and B. Zumino, Phys. Rev. 177 (1969) 2239; C. Callan, S. Coleman, J. Wess and B. Zumino, Phys. Rev. 177 (1969) 2247; M. Chanowitz, H. Georgi and M. Golden, Phys. Rev. Lett. 57 (1986) 2344.

18. This correlation, arising from the polarization of the top quark, is absent in the full event generators such as PYTHIA or HERWIG. The efficiency of applying this angular correlation to suppress the background, as done in (2.4), is higher if the minimum transverse momentum of the $b$ jet as described in (2.2) is lower. Therefore, combining the cuts (2.2) and (2.4) would guarantee the improvement of the signal-to-background ratio.

19. Applying the cuts of Ref. 10, we reproduced the result that for a $140 \mathrm{GeV}$ top quark the $t \bar{t}$ background rate is about the same as the signal rate.

20. The cross section of (2.1) is about $1.5 \mathrm{pb}$, but the true cross section is about $2 \mathrm{pb}$ after applying the prescription given in Ref. 12 .

21. B. Denby, preprint Fermilab-conf-92-121-E, 1992.

22. Based on the study done in "Physics at Fermilab in the 1990's", August 15-24, 1989, Breckenridge, Colorado, the expected $t \bar{t} \rightarrow e \nu+X$ signal rate 
in top search is only about 20 events with a $100 \mathrm{pb}^{-1}$ integrated luminosity and $\sqrt{S}=1.8 \mathrm{TeV}$ at the Tevatron.

\section{FIGURE CAPTIONS}

1) $m_{t}$ distribution for a $140 \mathrm{GeV}$ top quark at the Tevatron. It includes both the signal and the background events with $W^{ \pm} \rightarrow e^{ \pm}$or $\mu^{ \pm}$after the cuts $(2.2)$ and (2.4).

2) Same as Fig. 1 but with detector resolution effects as described in (2.5). 


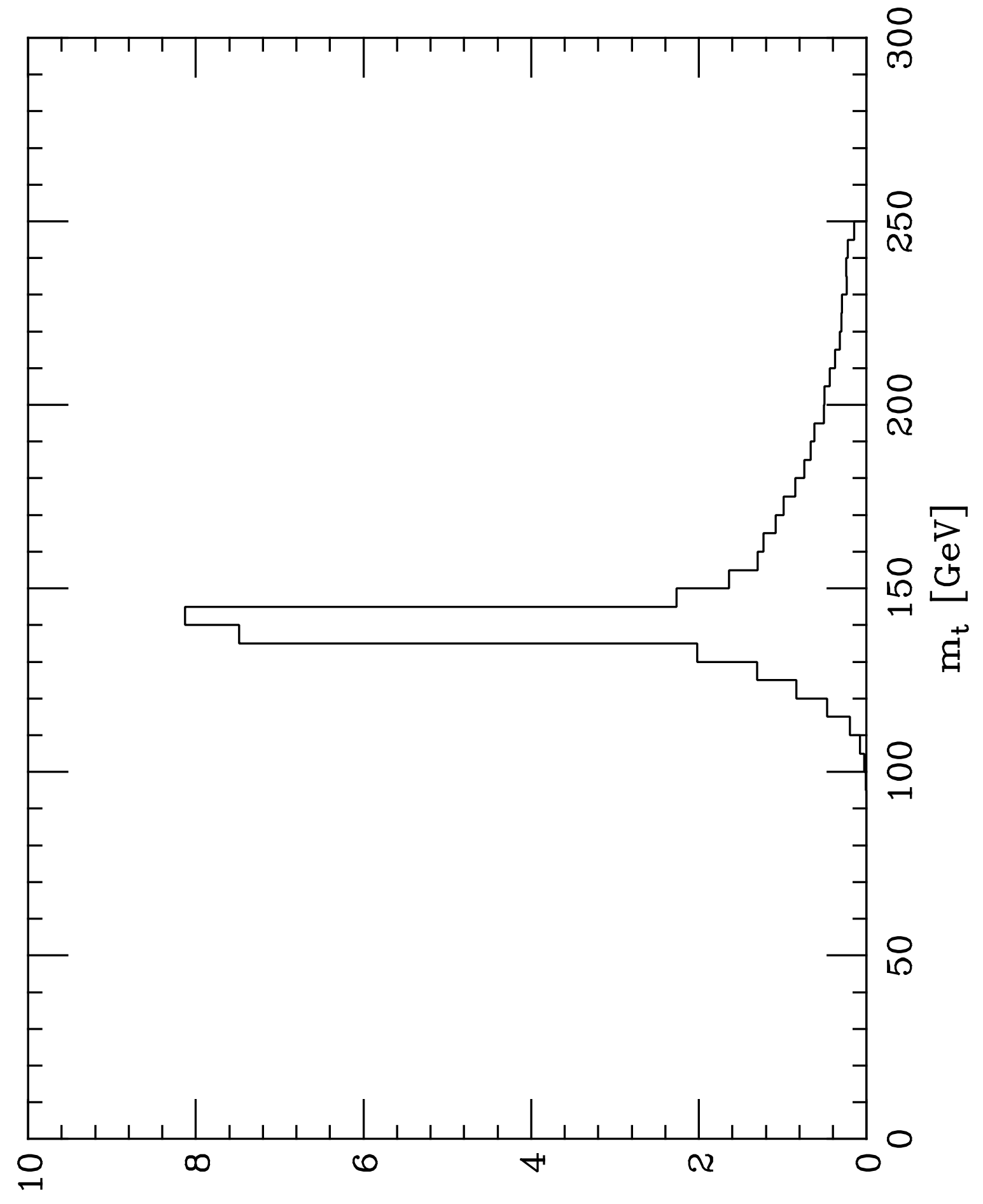

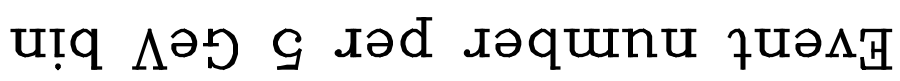




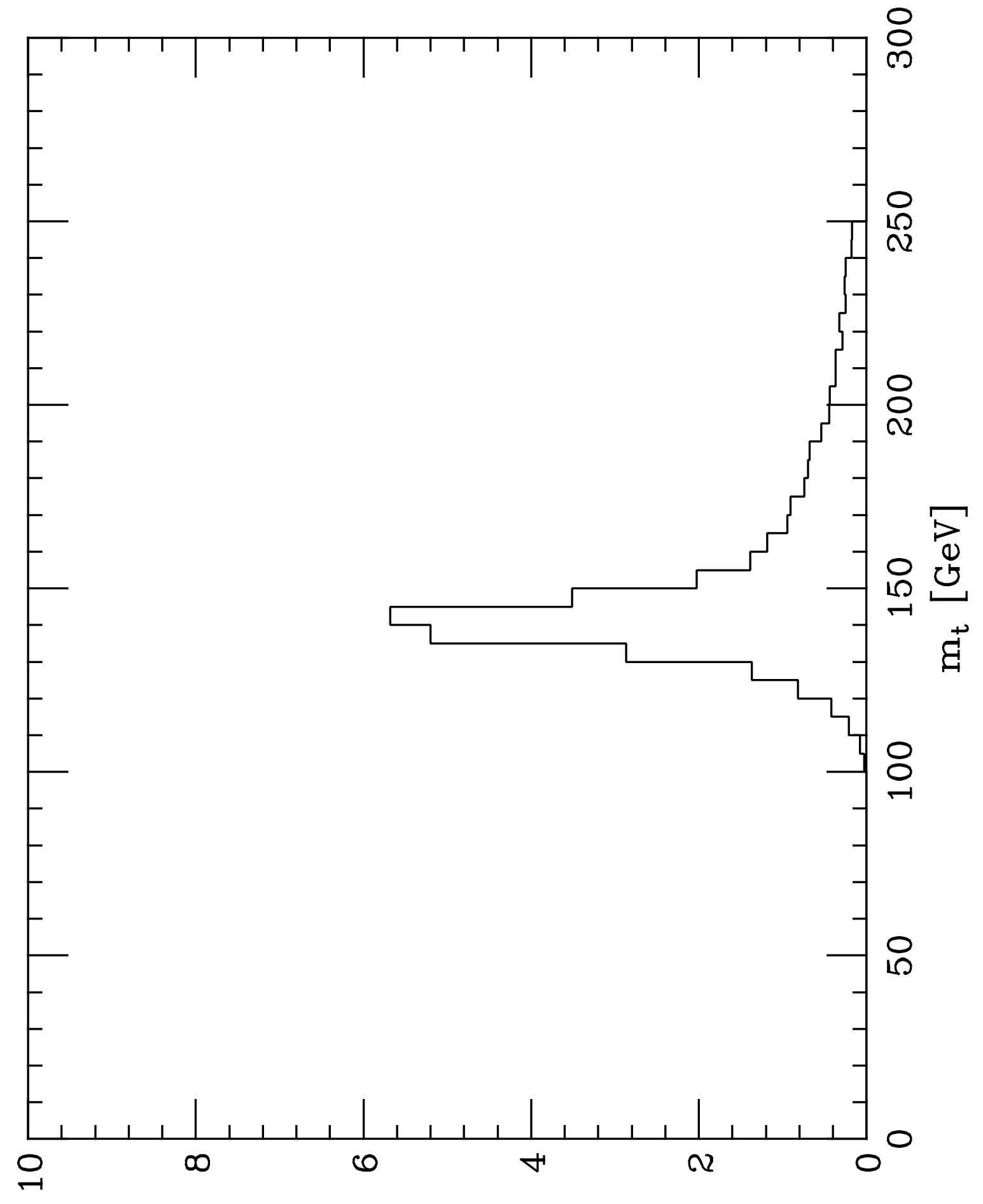

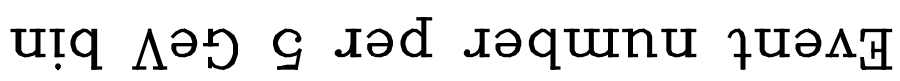

